\title{
Self Learning of ANFIS Inverse Control using Iterative Learning Technique
}

\author{
Kadhim H. Hassan \\ Basrah Technical College \\ Foundation of Technical \\ Education \\ Electrical Eng. Department
}

\begin{abstract}
This paper proposes an approach to tune an Adaptive Neuro Fuzzy Inference System (ANFIS) inverse controller using Iterative Learning Control (ILC). The control scheme consists of an ANFIS inverse model and learning control law. Direct ANFIS inverse controller may not guarantee satisfactory response due to different uncertainties associated with operating conditions and noisy training data. In this paper, the ILC makes a class of self tuning to the inputs of ANFIS inverse controller to minimize the overall system error so that the performance iteratively gets improved. The proposed scheme is simple, effective and lays out a unique tuning procedure for designing ANFIS inverse controller through ILC process.
\end{abstract}

\section{General Terms}

Intelligent Control, Nonlinear Control Systems.

\section{Keywords}

Iterative Learning Control, Adaptive Neuro Fuzzy Inference System, Inverse control, Labview

\section{INTRODUCTION}

The ANFIS is a very powerful approach for modeling nonlinear and complex systems with less input and output training data with quicker learning and high precision. It has the advantages of expert knowledge of fuzzy logic and learning capability of neural networks[1]. Since there introducing by Jang[2], the ANFIS has been widely used in several scientific and engineering applications. The ANFIS based inverse model control is a straightforward method for controller design in which the controller is the inverse of the plant. This approach only needs one learning task to find the plant inverse. To maintain high performance for complex and nonlinear processes, many challenges almost become apparent during actual application of direct ANFIS inverse controller such as model uncertainties, measurement noise and probable absent of training data. Several techniques have been adopted to overcome these challenges. In [3] a modified ANFIS inverse learning method is used by introducing a desired overall transfer model to obtain the required training data. Online parameter adaptation is used in inverse learning [4], that the inverse model of the plant is identified every time cycle. An adaptive ANFIS inverse controller is presented in [5], which the plant output is utilized by the adaptive algorithm to adjust the parameters of inverse controller to overcome the effects of nonlinear factors. So far these methods are complex and difficult in implementation.

This paper proposes a new combination structure of ANFIS inverse network and iterative learning control linked with the overall system error. to specify performance requirements.
Iterative Learning Control (ILC) is simple and very effect technique aimed at improving the tracking performance of a control system iteratively by using past experience in system operation. An ILC solves the optimization parametric problem by minimizing the objective function of specifying the performance of the system[6]. The basic idea behind the ILC is that the information obtained from previous trial is used to improve the reference input for the current trial. ILC is currently under comprehensive research investigation[6],[7], while an iterative learning tuner is presented in this paper to continuously adjusts the reference input of ANFIS inverse model iteratively to decrease the difference between the desired and actual output .of the system. The proposed approach is simple to implement with straightforward design and the structure dose not require any intermediate model of the plant as well as no online identification process needs for the inverse model parameters.

\section{OVERVIEW OF THE ANFIS}

Adaptive Neural Fuzzy Inference System (ANFIS) is fuzzy Sugeno model put in the framework to facilitate learning and adaptation procedure. Such network makes fuzzy logic more systematic and less relying on expert knowledge. The objective of ANFIS is to adjust the parameters of a fuzzy system by applying a learning procedure using input-output training data. A combination technique of least square algorithm and back propagation are used for training fuzzy inference system [8]. Basic architecture of ANFIS that has two inputs $x$ and $y$ and one output $f$ is shown in Fig.(1). Assume the rule base contains two Takagi-Sugeno if-then rules as follows:

Rule 1: If $x$ is $A_{1}$ and $y$ is $B_{1}$ Then $f_{l}=p_{1} x+q_{1} y+r_{I}$

Rule 2: If $x$ is $A_{2}$ and $y$ is $B_{2}$ Then $f_{2}=p_{2} x+q_{2} y+r_{2}$

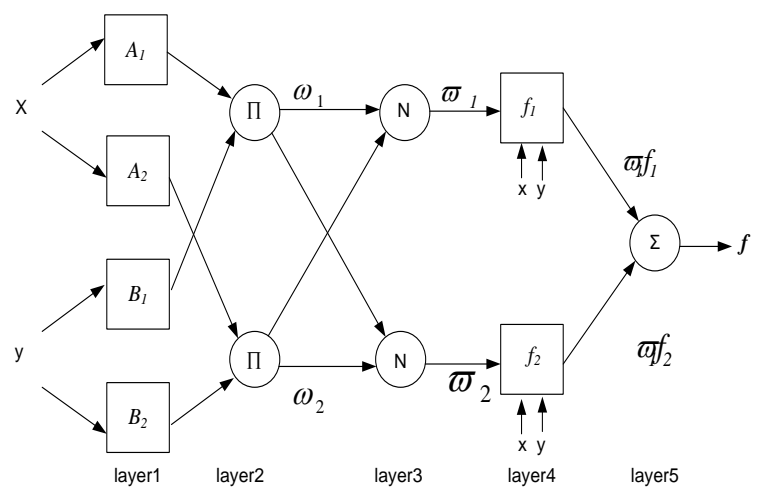

Fig. (1) Architecture of ANFIS ( two inputs-one-output Sugeno fuzzy model with two rules) 
ANFIS has five layers as shown in Fig.(1), square nodes are named adaptive nodes, to demonstrate that the parameters in these nodes are adjustable., while circle nodes, named fixed nodes, are to demonstrate that the Parameters are fixed. Then the node function in each layer is described below :

Layer 1: Every node $i$ in this layer is an adjustable node with node function :

$$
O_{1, i}=\mu_{A_{l}}(x) \quad i=1,2
$$

Where $x$ is the input to node $i$, and $A_{i}$ is the linguistic variable (large, small, etc.) . In other words, $O_{l, i}$ is the membership function of $A_{i}$ and it specifies the degree to the given $x$ satisfies the $A_{i}$ quantifier. We choose $\mu_{A_{l}}(x)$ to be a bell-shaped function with maximum equal to 1 and minimum equal to zero such as :

$$
\mu_{A_{i}}(x)=\frac{1}{1+\left(\frac{x-c_{i}}{a_{i}}\right)^{2 b_{i}}}
$$

Where $\left(a_{i}, b_{i}, c_{i}\right)$ is the parameter set. As the values of these parameters change, the bell-shaped vary accordingly

Layer 2: node $i$ in this layer is a fixed node, with the node function to multiply input signals whose output is the product of all the incoming signals:

$$
O_{2, i}=\omega_{i}=\mu_{A_{i}}(x) \times \mu_{B_{i}}(y) \quad i=1,2
$$

Every node output represents the firing strength of a fuzzy rule.

Layer 3: Each node $i$ in this layer is a fixed node labelled $\mathrm{N}$. The ith node computes the ratio of the ith rule's firing strength to the sum of all rule's strengths .

$$
O_{3, i}=\varpi_{i}=\frac{\omega_{i}}{\omega_{1}+\omega_{2}} \quad i=1,2
$$

The Output of this layer is called " normalized firing strength"

Layer 4: Every node $i$ in this layer is an adaptive node of a function :

$$
O_{4, i}=\varpi_{i} f_{i}=\varpi_{i}\left(p_{i} x+q_{i} y+r_{i}\right)
$$

$\omega_{i}$ : is a normalized firing strength from layer 3. $\left(p_{i}, q_{i}, r_{i}\right)$ is the parameter set of this node. Which is referred to the consequent parameters.

Layer 5: The single node in this layer is a fixed node labelled $\sum$, that calculates the overall output as the summation of incoming signals:

$$
O_{5}=\sum_{i} \varpi_{i} f_{i}=\frac{\sum_{i} \omega_{i} f_{i}}{\sum_{i} \omega_{i}}=\frac{\omega_{1} f_{1}+\omega_{2} f_{2}}{\omega_{1}+\omega_{2}}
$$

The ANFIS architecture has been constructed, so we can apply a learning procedure to find its parameters for achieving a minimal error measurement.. A hybrid learning algorithm is adopted as follows:

In the forward pass, node outputs go forwards until layer four and the consequent parameters are identified by using the Least Square technique while In backward pass, the error signals propagate backward and the set of premise parameters is updated by Gradient Descent method. The advantage of this hybrid learning versus the pure gradient descent method, the rapid convergence to the global minimum is guaranteed.

\section{ANFIS INVERSE TRAINING}

The structure of the ANFIS inverse based control system is mainly consist of the ANFIS inverse network, that acts as a controller to generate the control action, is placed in series with the plant. To obtain the ANFIS inverse model of the plant, the training process is shown in Fig.(2), for the training, the inputoutput data set is used to reflect input-output characteristics of the plant .

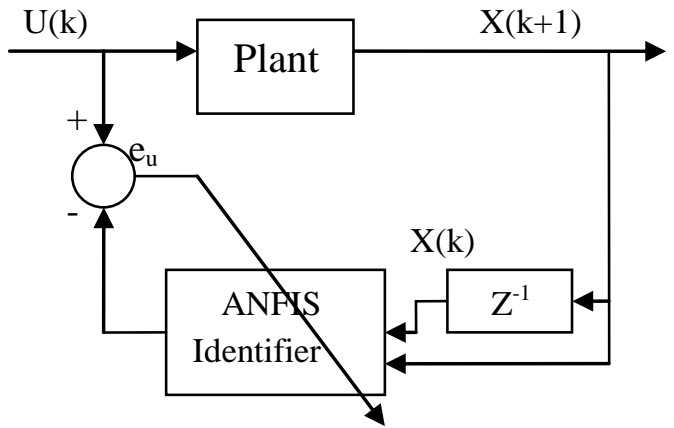

Fig(2) The training process of ANFIS based on inverse plant model

Using the training data set :

$$
\left(X^{N}(k), X(k+1)^{N} ; U^{N}\right)
$$

Where $\mathrm{N}$ is the number of training data. The ANFIS network can be trained by least square estimation method to minimize the error cost function $\mathrm{E}$ defined by:

$$
E=\sum_{i=1}^{N}\left(U_{m}-O_{m}^{i}\right)^{2}
$$

Where $U_{m}$ is the control input signal and $O_{m}^{i}$ is the corresponding actual output of the ANFIS identifier of the m-th component. 


\section{THE PROPOSED APPROACH}

The Iterative learning tuner is designed to improve the tracking performance of ANFIS inverse control which has a repeated desired task over a finite interval. The inputs, outputs and states of the system are propagated along two independent dimensions. One indicated by variable $\mathrm{j}$ describes the variables in term of time history. and the other process $\mathrm{k}$ reflects the learning iteration. For example $y(j, k)$ represents the jth item of the output in the kth learning cycle , in which $j=1, \ldots, n$ and $k=1, \ldots ., \infty$. A basic block diagram of the proposed structure is shown in Fig.(3).

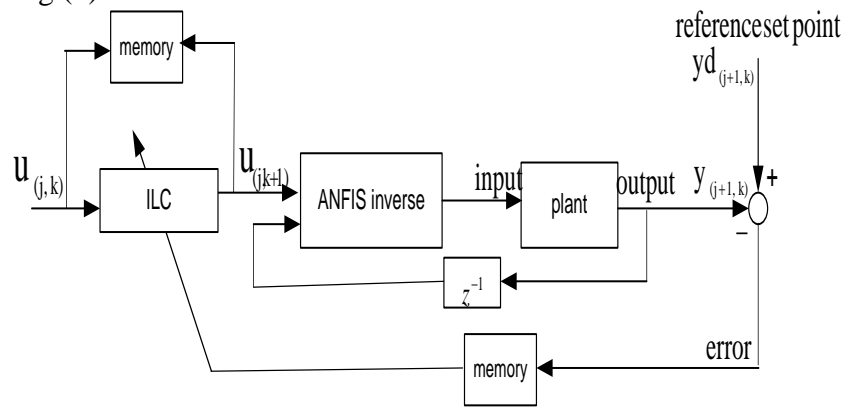

Fig.(3) The block diagram of the proposed controller

The following learning law is used in the proposed :structure to continuously adjusts the control input :

$$
\begin{aligned}
& u_{(j, k+1)}=u_{(j, k)}+\phi e_{(j, k)} \\
& e_{(j, k)}=y d_{(j, k)}-y_{(j, k)}
\end{aligned}
$$

Where: $\boldsymbol{\phi}$ is the learning step which ensured that:

$$
\begin{aligned}
& y_{(j, k)} \rightarrow y d_{(j, k)} \text { as } k \rightarrow \infty \\
& u_{(j, k)}: \text { the input at k-th trial } \\
& y d_{(j, k)}: \text { the desired output } \\
& y_{(j, k)}: \text { the actual plant output }
\end{aligned}
$$

$$
e_{(j, k)}: \text { the overall system error }
$$

In the proposed controller, the ANFIS inverse controller is updated at each trial according to the actual output of the plant under control which can manage model error and uncertainties by minimizing the error of the system then ILC improves the control system iteratively to a better performance with a better precise.

To ensure the stability and convergence of the proposed method. The following assumptions are made:

1-The desired output signal $y d_{(j, k)}$ is repeatable over a finite time interval.

2-The system is stable under the ANFIS inverse control before the iterative learning is introduced.

3-The value of control input signal $u_{(j, k)}$ is bounded.

\section{SIMULATION AND RESULTS}

The proposed scheme presented in this paper is tested through simulation to validate the performance of the proposed controller. The simulation involves a model of coupled tank system, when a two tanks are connected together.

$$
f_{1}-f_{2}=\frac{d V_{2}}{d t}=A \frac{d h_{2}}{d t}
$$

Where:

$f_{2}$ : The out flow rate of tank2

$h_{2}$ : The height of tank $2 ; V_{2}$ :The volume of tank 2 .

The inter-tank holes are assumed to behave as orifices, then:

$$
\begin{aligned}
& f_{1}=\sqrt{c_{d 1} a_{1} 2 g\left(h_{1}-h_{2}\right)} \\
& f_{2}=\sqrt{c_{d 2} a_{2} 2 g\left(h_{2}-h_{3}\right)}
\end{aligned}
$$

\subsection{Simulation example Consider a tanks system as shown in Fig.(4).}




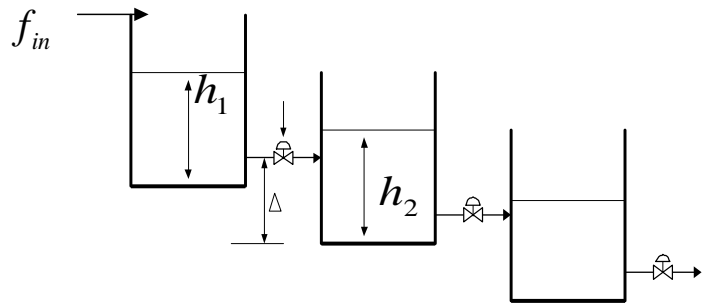

Fig(4) The coupled tank system

The aim is to design a level controller of the second tank $\left(h_{2}\right)$

To simulate the system, a dynamic model is derived. For the first tank the flow balance equation is:

$$
f_{\text {in }}-f_{1}=\frac{d V_{1}}{d t}=A \frac{d h_{1}}{d t}
$$

Where $f_{\text {in }}$ is the fed flow rate

$$
\begin{aligned}
& f_{1} \text { :The flow rate from tank } 1 \text { to tank2 } \\
& V_{1}: \text { The volume of tank1 } \\
& h_{1} \text { The height of tank } 1
\end{aligned}
$$

\section{$\boldsymbol{A}$ :The cross section area of tank 1 and tank 2}

For the second tank:

$a_{1}:$ Cross section area of orifice 1

$a_{2}$ : Cross section area of orifice 2

$c d_{1}, c d_{2}:$ Discharge coefficient

$h_{3}$ Height of drain tap

$g$ : Gravitation constant

\section{Where:}

Labview based software tool is used for simulation. The block diagram of the Labview platform is shown in Fig.(5). We successfully integrate Matlab into Labview to design the ANFIS model through Matlab script node available in Labview.

The physical parameters of the coupled tank system are given below: $h_{1}=h_{2}=1 m, A=0.01 m^{2}$
The orifice area of the first tank is $a_{1}=0.15 \mathrm{~m}^{2}$ and the Orifice area os tank2 is : $a_{2}=0.125 \mathrm{~m}^{2}$. The maximum fed flow rate is: $f_{\text {in }}=0.5$ litter $/ \mathrm{sec}, \mathrm{g}=9.81 \mathrm{~m} / \mathrm{sec} 2$; $c d_{1}=c d_{2}=1$ and $h_{3}=\mathrm{O}$ The states are the liquid level in tank 1 and level in tank 2. If the control input is the flow rate $f_{\text {in }}$, the variable to be controlled is the second state tank level $\boldsymbol{h}_{2}$.

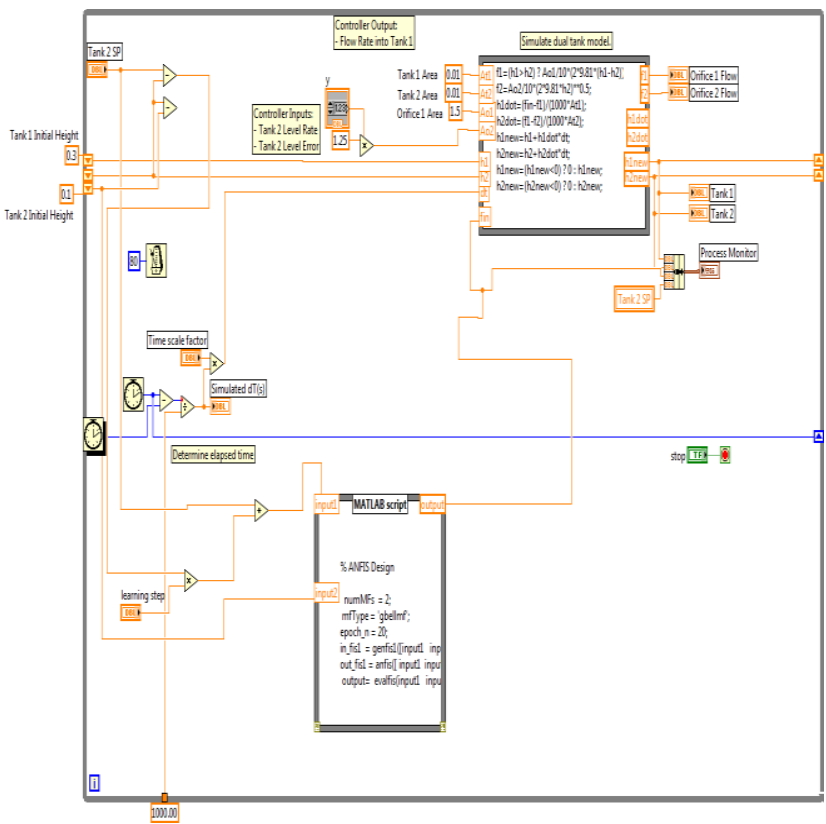

\section{FIG.(5) The Labview block diagram of the proposed} controller

\subsection{Controller Design and Results}

In the simulation, the ANFIS inverse network is designed based on first order Sugeno fuzzy model so the consequent part of the fuzzy rules is a linear equation with generalized bell-shaped membership functions are chosen of the inputs, which contain three fitting parameters ; centre and half of the width and slop. Two membership functions are chosen on each input.

The initialization of primes parameters of ANFIS network are set so that the centers of the membership functions are equally spaced entire the range of the input variable, where the initial value of consequent parameters set is assumed zero. P-type learning law is used with learning step of 0.15 . The collected input-output data of the coupled tank is applied to train the ANFIS to do the inverse of the tank system. The training data including the input flow rate of the second tank and the corresponding level with the delayed level signal. The trained model then used as inverse controller in the proposed approach as shown in the structure Fig.(3).

For the sake of comparison, a fuzzy logic controller for the coupled tank system is designed based on Labview fuzzy toolkit[9]. The simulation results of time response characteristic due to step set point tracking from initial level to $0.5 \mathrm{~m}$ is shown in $\operatorname{Fig}(6 . a, b)$ for the proposed controller and fuzzy logic 
controller respectively. The proposed controller effectively reaches the set point, it has no steady state error with faster response as compared with the fuzzy controller. Then the set point for tank 2 is changed up and down between $0.1 \mathrm{~m}$ and $0.5 \mathrm{~m}$ as shown in Fig.(7.a,b). It can be seen clearly that the proposed controller successfully fast tracks the demands of level set point.

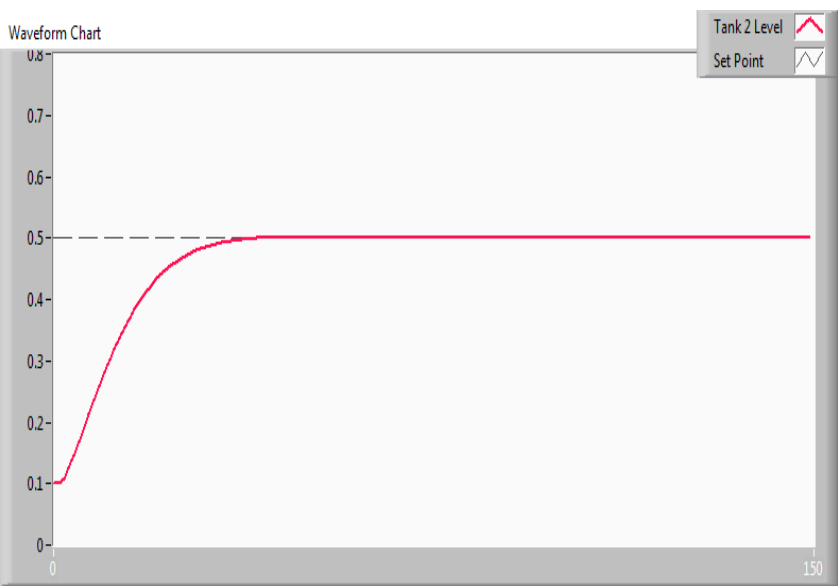

(a)

(a) The proposed controller

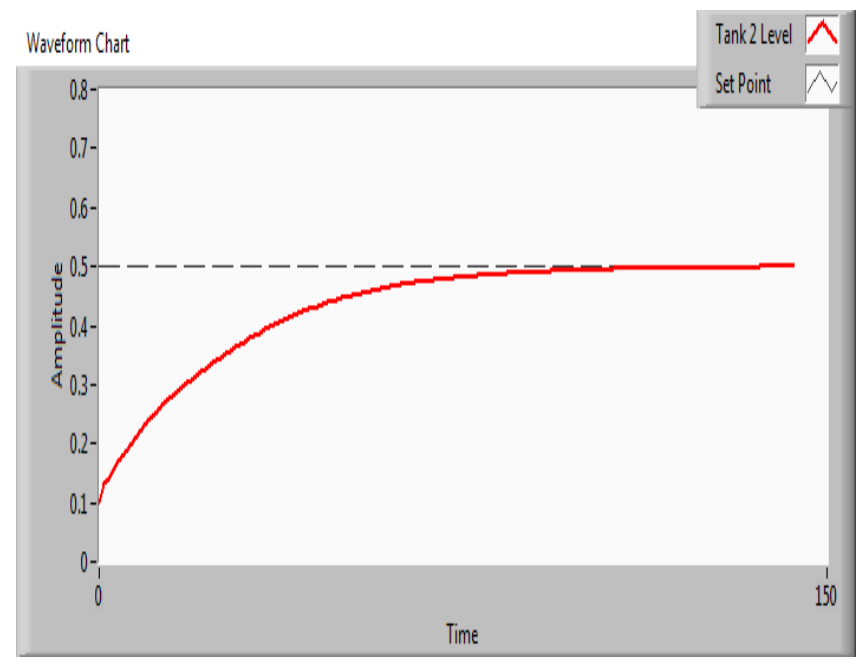

(b) Fuzzy Logic Controller

Fig(6) Time response characteristic for step set point

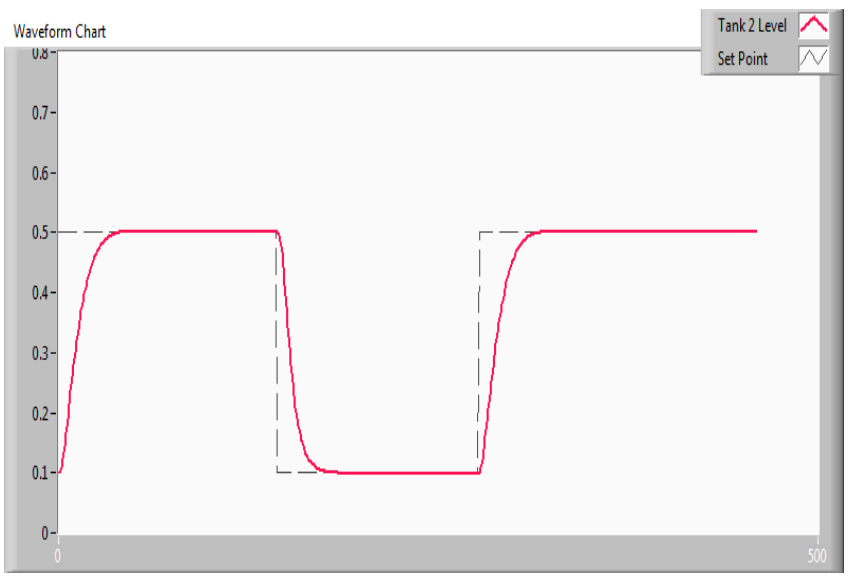

(a) The proposed Controller

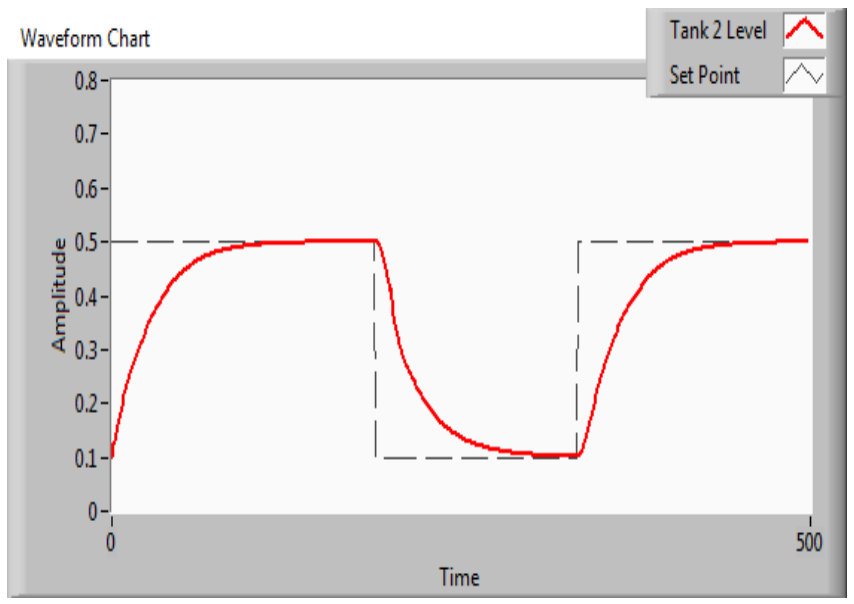

(b) Fuzzy logic controller

Fig.(7) Multiple set point tracking characteristic

\section{CONCLUSION}

In this paper, a new design structure which combines the ANFIS inverse model and ILC was proposed in which the inputs of the ANFIS controller is adaptively adjusted through iterative learning law. The ANFIS is trained to model the inverse dynamics of process from measurement data. It is difficult to obtain the exact dynamic characteristic through direct training process hence in this research we have used iterative learning tuner so that the overall system error is online minimized. The simulation results show the effectively of the proposed scheme. 


\section{REFERENCES}

[1] K. Guney and N. Sankaya " Adaptive Neural Fuzzy Inference System for Computing the Physical Dimensions of Electrically Thin and Thick Rectangular Microstrip Antennas" Int. J. of Infrared and Millimeter Waves Vol.27, No.2, Feb. 2006.

[2] J.S.R. Jang " Adaptive Network- Based Fuzzy Inference. System' IEEE Trans. On Systems Man. and Cybemetics, Vol.23, No.3, pp 665-685, 1993

[3] J. D. Wang, N. Y. Chen ' Modified ANFIS Inverse Learning for Servo Motor Speed Controller Design", Proceedings of CACS Automatic Control Conference Taiwan, Nov. 2005.

[4] M. A. Denal, F. Palis and A. Zeghbib " ANFIS Based Modeling and Control of Non-linear systems: A tutorial", IEEE International Conference on Systems, Man. and Cybemetics, pp. 3433-3438, 2004.
[5] C. Tao, H. Ruicai and P. Feng " Adaptive Inverse Control of Linear Motor Based on ANFIS'" Proceedings of the $29^{\text {th }}$ Chinese Control Conference, China July 2010.

[6] D. A. Bristow, M. Tharayil and A. G. Alleyne, "A Survey of Iterative Learning Control" IEEE Control Syst. Mag. Vol.26, No.3, pp 96-114, 2006

[7] H. Sung, Y. Q. Chen and K. Moore " Iterative Learning Control : Brief Survey and Categorization" IEEE Transaction on Systems, Man. and Cybernetics-Part C: applications and reviews, Vol. 37, No.6, November 2007.

[8] K. Assaleh, "Extraction of Fetal Electrocardiogram Using Adaptive Neuro-Fuzzy Inference System" IEEE Transactions on Biomedical Engineering, Vol.54, No.1, PP. 59-68, 2007

[9] Labview PID and Fuzzy Logic Toolkit User Manual National Instrument, USA June 2009. 